\title{
Sanjin tablet combined with antibiotics for treating patients with acute lower urinary tract infections: A meta-analysis and GRADE evidence profile
}

\author{
JIAN LYU, YANMING XIE, MENGHUA SUN, CHENG ZHANG and LIANXIN WANG
}

Institute of Basic Research in Clinical Medicine, China Academy of Chinese Medical Sciences, Beijing 100700, P.R. China

Received June 3, 2019; Accepted November 8, 2019

DOI: $10.3892 /$ etm.2019.8252

\begin{abstract}
Numerous clinical studies have evaluated the performance of Sanjin tablets (SJTs) in the treatment of acute lower urinary tract infections (ALUTIs) in China. The present meta-analysis aimed to determine the efficacy and safety of SJT combined with antibiotics for the treatment of patients with ALUTIs and to evaluate the quality of evidence. The Cochrane Library, PubMed, EMBASE, Web of Science, Chinese BioMedical Database, China National Knowledge Infrastructure, WanFang and VIP databases were searched for entries added between inception and December 2018 to identify relevant randomized controlled trials (RCTs). The Cochrane risk-of-bias tool and Consolidated Standards of Reporting Trials for Traditional Chinese Medicine were used for assessing the methodological quality and reporting quality of eligible studies, respectively. Meta-analysis and quality of evidence assessment were performed with RevMan 5.3 and Grading of Recommendations, Assessment, Development and Evaluations (GRADE), respectively. A total of 8 RCTs comprising 790 patients with ALUTIs were included in the present meta-analysis. The cure rate of SJTs combined with gatifloxacin tablets (GTs) was higher than that of GTs alone [relative ratio $(\mathrm{RR})=1.30,95 \% \mathrm{CI}=1.07-1.57, \mathrm{P}=0.009]$ ]. The cure rate of SJTs combined with levofloxacin tablets (LTs) was higher than that of LTs alone $(\mathrm{RR}=1.13,95 \% \mathrm{CI}=1.04-1.24$, $\mathrm{P}=0.006)$. SJTs combined with LTs was better in improving the total effective rate than LTs alone $(\mathrm{RR}=1.11,95 \% \mathrm{CI}=1.03-1.19$,
\end{abstract}

Correspondence to: Professor Yanming Xie, Institute of Basic Research in Clinical Medicine, China Academy of Chinese Medical Sciences, 16 Nanxiao Street, Beijing 100700, P.R. China E-mail: ktzu2018@163.com

Abbreviations: SJT, Sanjin tablet; ALUTI, acute lower urinary tract infection; RCT, randomized controlled trial; RR, risk ratio; ADE, adverse event; ADR, adverse reaction; LT, levofloxacin tablet; GT, gatifloxacin tablet; OT, oxyfluoxacin tablet; GRADE, Grading of Recommendations, Assessment, Development and Evaluations

Key words: acute lower urinary tract infection, Sanjin tablet, meta-analysis, Grading of Recommendations Assessment Development and Evaluations
$\mathrm{P}=0.005)$. The recurrence rate for SJTs combined with antibiotics was lower than that associated with antibiotics alone ( $\mathrm{RR}=0.35,95 \% \mathrm{CI}=0.13-0.97, \mathrm{P}=0.04)$. The bacterial clearance rate achieved with SJTs combined with antibiotics was higher than that obtained with antibiotics alone $(\mathrm{R} R=1.41$, 95\% CI=1.09-1.84, P=0.009). The present meta-analysis demonstrated that, compared with the effects of antibiotics treatment, SJTs combined with antibiotics improved the cure rate, total effective rate and bacterial clearance rate, and decreased the recurrence rate. In addition, no serious adverse reactions were observed in patients with ALUTIs. However, the GRADE quality of evidence was low. Thus, further large-scale and rigorously designed clinical trials are required to improve the quality of evidence.

\section{Introduction}

Urinary tract infections (UTIs) are among the most common bacterial infections acquired in the general population and in hospitals (1). A variety of pathogens, including bacteria, fungi, mycoplasma, chlamydia and viruses, may cause UTIs, which are mainly characterized by painful, frequent and urgent urination, as well as urethral burning (2). In the USA, $>7$ million outpatients and $\sim 1$ million in-patients with UTIs are encountered in the clinic each year (3), while $\sim 150$ million patients are diagnosed with UTIs worldwide each year (4). UTIs, which may cause septic shock, rank third among all diseases that may lead to death as a result of infection (5). Gram-negative bacteria are the major cause of community-acquired and hospital-acquired UTIs $(4,6)$. At present, antibiotics are the major treatment for UTIs, but not all patients benefit from them. Abuse of antibiotics markedly increases the drug resistance of bacteria, reduces the clinical efficacy of antibiotics and increases the recurrence rate of bacterial infection, which wastes medical resources and reduces the quality of life of patients (7). Multiple Chinese traditional medicines have been demonstrated to exert bacteriostatic effects on pathogenic microorganisms, and may therefore inhibit or destroy the formation of toxic substances $(8,9)$. A test of antibacterial properties in mice revealed that Sanjin tablet (SJTs) has a marked bacteriostatic effects (10).

SJT is composed of five types of Chinese herbal medicines: Baqia (Chinaroot Greenbrier Rhizome), Jinyinggen (Root of Cherokee Rose), Yangkaikou (Fruit of Fiverleaf 
Akebia), Jinshateng (Lygodii Herba) and Jixuecao (Asiatic Pennywort Herb), which were recorded in the Chinese Pharmacopoeia 2015 (11). According to the concepts of Chinese Traditional Medicine, the herbal components have the following properties: Jinyinggen is acerb and neutral in nature, and is able to control nocturnal emission and astringent intestine (12). Baqia is bitter and neutral in nature; it is able to alleviate rheumatism, promote blood circulation, detoxicate, relieve convulsion and calm endogenous wind (13). Jinshateng is slightly sweet and cold-natured, and is able to clear heat, detoxicate and remove dampness (14). These three herbs are monarch drugs (major components) in the prescription, which have an enhancing effect on the functions of anti-inflammation, dehumidification and detoxification (15). The above three drugs supplemented with Yangkaikou and Jixuecao exhibit enhanced effects against UTIs (16,17).

Previous clinical studies have demonstrated that SJTs are able to reduce the symptoms of chronic UTIs, the number of recurrences and the secretory level of urinary soluble interleukin (IL)-2 receptor, IL-6 and IL-8 in patients with chronic nephropyelitis (10). Electron microscopy revealed that SJTs is able to make the flagella of Escherichia coli drop (18). Lower UTIs mainly comprise cystitis and urethritis, which have high incidence and recurrence rates. In recent years, numerous clinical studies have explored the efficacy and safety of SJTs combined with antibiotics in the treatment of acute lower UTIs (ALUTIs) in China. However, there is no meta-analysis on SJTs combined with antibiotics for the treatment of ALUTIs. To the best of our knowledge, the present study is the first comprehensive systematic review that determined the efficacy and safety of SJTs combined with antibiotics for the treatment of ALUTIs. The Grading of Recommendations, Assessment, Development and Evaluations (GRADE) system $(19,20)$ was used to evaluate the quality of evidence of the key outcomes of the present meta-analysis, which provides a basis and serves as a reference for clinical practice guidance.

\section{Materials and methods}

Search strategy. Electronic databases, including PubMed, EMBASE, Cochrane Library, Web of Science, China National Knowledge Infrastructure, Chinese BioMedical Database, WanFang Database and VIP Database (VIP) were systematically searched for entries added between inception and December 2018. The following search terms were used separately or in combination: 'Sanjin' or 'Sanjin tablet' AND 'acute lower urinary tract infection' or 'acute lower UTI'.

Selection criteria. Studies were selected according to the following inclusion criteria: i) Participants were diagnosed with ALUTIs; ii) the study was performed as a randomized controlled trial (RCT); iii) efficacy of SJT combined with antibiotics vs. antibiotics, including levofloxacin tablets (LTs), gatifloxacin tablets (GTs) and ofloxacin tablets (OTs); iv) primary outcomes were the cure rate (i.e. the symptoms disappeared and the leukocyte levels in the urine returned to normal after treatment) and the recurrence rate (i.e. the symptoms of the patients reappeared or their urine leukocyte value increased again); and v) secondary outcomes included the total effective rate (i.e. the symptoms partially disappeared or the value of urine leukocytes was reduced but did not return to normal after treatment), bacterial clearance rate (the original infected part of the specimen did not regenerate after treatment), incidence of adverse reactions (ADRs) and any adverse events (ADEs), including headache, stomach ache, stomach discomfort, mild nausea, skin rash and dizziness.

The exclusion criteria were as follows: i) Insufficient data (miscalculation or missing data); ii) the full text was not available; iii) duplicated data; and iv) the intervention included other Chinese drugs, acupuncture and massage (including a proprietary Chinese drug, Traditional Chinese Medicine extract injection, decoction, auricular points and other Traditional Chinese Medicine methods as auxiliary treatment).

Literature screening. EndNote (v. 8.1.11010; Clarivate Analytics) was used to identify duplicates among the studies retrieved. After reading the titles and abstracts of the studies obtained for preliminary screening, those articles that did not meet the inclusion criteria were excluded. The full text of the articles that potentially met the inclusion criteria was further screened to determine whether they should be included in the present study. The list of references of the studies retrieved were also checked to identify any further studies. The literature was independently screened by two researchers (JL and MS) according to the inclusion/exclusion criteria. Any disagreement between the reviewers was resolved by consulting a third party (LW and YX).

Data extraction. Two independent researchers, namely JL and MS, were responsible for data extraction and any disagreements were resolved by a third author (YX). The number of events and the total number of patients in each group were extracted from binary outcomes. The mean, standard deviation and sample size for each group were extracted or inputted from continuous outcomes. The data extracted included the following: Name of the first author, year of publication, method of randomization, number of patients, sex and age in the comparison groups, as well as the total number of patients, the drug dose and duration of treatment in the comparison groups, primary and secondary outcomes, and any ADEs or ADRs. When the study had $\geq 1$ common intervention group, the method recommended by the Cochrane Collaboration was followed, i.e. grouping and merging, and conversion of the multi-arm trial into a 2-arm trial (21).

Quality assessment. The Cochrane Handbook for Systematic Reviews of Interventions 5.1 'bias risk assessment' tool was used to assess quality in seven domains: Random sequence generation; allocation concealment; blinding of participants and personnel; blinding of outcome assessment; incomplete outcome data; selective outcome reporting; and other bias (21). The risk of bias was classified as low, high or unclear. JL and CZ were responsible for independent assessment of quality and any disagreements were resolved by a third author (YX).

Statistical analysis. RevMan 5.3, provided by the Cochrane Collaboration Network, was used for meta-analysis. The relative ratio (RR) and 95\% CI were used for binary outcomes and the weighted mean difference and $95 \%$ CIs were used 
for continuous outcomes. The $\mathrm{I}^{2}$ statistic was used to assess heterogeneity; if $\mathrm{I}^{2}<50 \%$, the statistical homogeneity was considered to be acceptable and the fixed-effects model was used, and if $\mathrm{I}^{2} \geq 50 \%$, a significant statistical heterogeneity was assumed and the random-effects model was adopted. If the study was not suitable for meta-analysis, a descriptive analysis was performed. A funnel plot was used to detect publication bias.

Subgroup and sensitivity analyses. In order to address heterogeneity, subgroup analysis was performed, which focused on the cure rate of the different interventions. Interventions included SJTs combined with GTs vs. GTs and SJTs combined with LTs vs. LTs. Sensitivity analysis was performed to assess the influence of a single study on the overall pooled estimate by removing one study at a time.

GRADE assessment. The GRADE system was used to classify the quality of evidence of the cure rate, total effective rate, recurrence rate and incidence of ADRs. According to the GRADE classification method, the RCTs are initially classified as studies with the highest quality of evidence and their quality was then decreased based on 5 factors (risk of bias, inconsistency, indirectness, imprecision and publication bias), and the quality of the final evidence was classified as high, moderate, low and very low $(19,20)$.

\section{Results}

Literature search results. The initial literature search identified 64 studies. After duplicates among different databases were removed by using EndNote, the title and abstract of the studies were read, and the inclusion and exclusion criteria were combined, and 36 articles were selected for evaluation of their full text. A total of 28 articles were excluded for not being RCTs ( $n=16)$, not correctly performing intervention measures $(n=3)$, unavailability of data $(n=5)$ and containing duplicate or incorrect data $(n=4)$. A total of 8 trials were eventually selected for inclusion in the present meta-analysis, all of which were published in Chinese (22-29). The selection process of the studies is presented in Fig. 1.

Characteristics of the studies included. A total of 8 studies were included in the present meta-analysis, all of which described that the baseline values of the experimental and control groups were comparable. All patients included in the present meta-analysis underwent a urine culture test at the time-point of diagnosis. After treatment, a urine culture test was performed to determine the curative effect. A change in urine bacterial culture for the same strain from positive to negative was considered to indicate cure. In total, 3 studies were 3 -arm trials $(22,23,28)$, and 2 of them were double-blinded and double-simulated studies (22,23). After grouping and combining these 3 -arm trials, the interventions were as follows: SJTs vs. antibiotics and SJTs combined with antibiotics vs. antibiotics. The interventions of the other 5 studies were SJTs combined with antibiotics vs. antibiotics. Other conventional and adjuvant treatments were not mentioned in any of the studies. The total sample size was 790 cases, including 405 cases in the experimental group and
385 cases in the control group. The average daily dose of SJTs was 12 pills (3.48 g). In 5 studies, patients received treatment for 7 days, while in other studies, patients received treatment for 3 (27), 5 (17) and 3-15 days (26). A total of 7 trials reported on ADEs/ADRs. The characteristics of the 8 trials included are listed in Table I.

Methodological quality. None of the 8 studies reported on the study protocol, sample size estimates, randomization, blinding or allocation concealment. A total of 6 studies mentioned that the patients were followed up after treatment to evaluate recurrence, but only 3 studies had followed up data $(17,27,28)$. In total, 6 studies reported on withdrawals and loss to follow-up, but no intention analysis was performed $(22-25,27,28)$. One study did not fully report on pre-specified outcomes and exhibited selective reporting of results (24). The results of the quality assessment of the studies included are provided in Figs. 2 and 3.

\section{Meta-analysis}

Cure rate. The cure rate was assessed for a total of 790 patients across all of the studies included. In total, 7 studies $(17,22-25,27,28)$ were divided into 2 subgroups according to the different interventions and the homogeneity was good in each subgroup $\left(\mathrm{P}=0.73 / 0.72, \mathrm{I}^{2}=0\right)$. The fixed-effects model was used for the meta-analysis. The results indicated that the cure rate of SJTs combined with GTs was higher than that of GTs alone $(\mathrm{RR}=1.30,95 \% \mathrm{CI}=1.07-1.57$, $\mathrm{P}=0.009$; Fig. 4), and the cure rate of SJTs combined with LTs was higher than that of LTs alone $(\mathrm{RR}=1.13,95 \% \mathrm{CI}=1.04-1.24$, $\mathrm{P}=0.006$; Fig. 4). One study (26) performed a descriptive analysis and suggested that the cure rate of SJTs combined with OT was higher than that of OT alone $(\mathrm{RR}=1.38,95 \% \mathrm{CI}=1.03-1.84$, $\mathrm{P}<0.03)$.

Total effective rate. A total of 510 patients from 5 studies were assessed for the total effective rate. The intervention of 3 studies consisted of SJTs combined with LTs vs. LTs alone $(22,27,28)$. The heterogeneity test indicated that the fixed-effects model was appropriate for use $\left(\mathrm{P}=0.25, \mathrm{I}^{2}=29 \%\right)$. Meta-analysis demonstrated that the total effective rate of SJTs combined with LTs was higher than that of LTs alone ( $R R=1.11,95 \% \mathrm{CI}=1.03-1.19, \mathrm{P}=0.005$; Fig. 5). Analysis of the data of one study (17) suggested that the effective rate of SJTs combined with GTs was higher than that of GTs alone $(\mathrm{RR}=1.31,95 \% \mathrm{CI}=1.03-1.67, \mathrm{P}=0.03)$. One study (26) suggested that the total effective rate of SJTs combined with OT and that of OTs alone was not significantly different $(\mathrm{RR}=1.16,95 \% \mathrm{CI}=0.95-1.41 ; \mathrm{P}=0.14)$.

Recurrence rate. A total of 3 studies comprising 201 patients were assessed regarding the recurrence rate $(17,27,28)$. The interventions were SJTs combined with GTs vs. GTs and SJTs combined with LTs vs. LTs. In order to comprehensively evaluate the effect of SJT combined with antibiotics, the 3 interventions were classified as SJT combined with antibiotics vs. antibiotics alone for combined analysis. The homogeneity of the 3 studies was good $\left(\mathrm{P}=0.69, \mathrm{I}^{2}=0 \%\right)$ and the fixed-effects model was used for the meta-analysis. Statistical analysis indicated that the recurrence rate of SJT combined with 


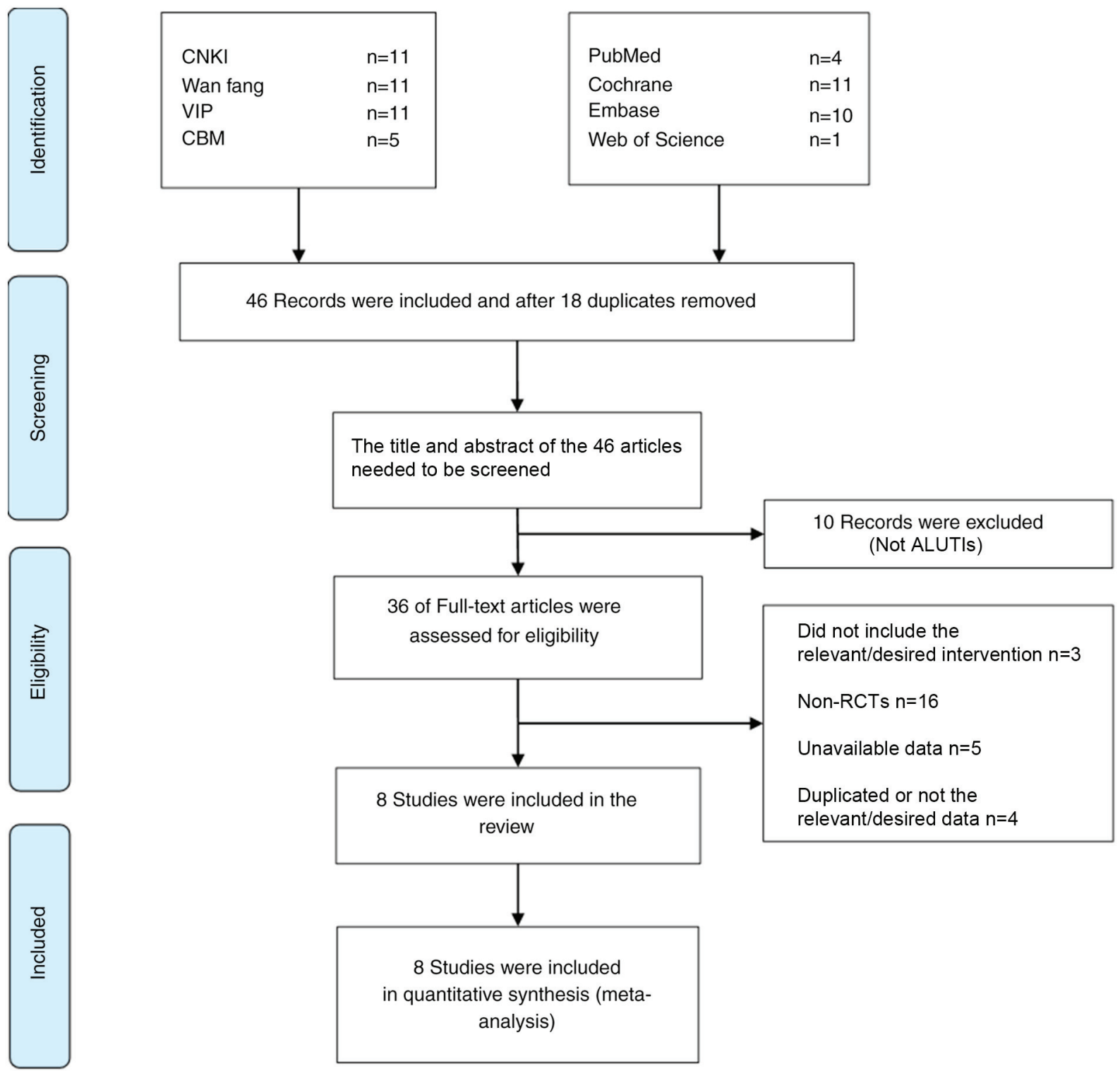

Figure 1. Preferred Reporting Items for Systematic Reviews and Meta-Analyses flow diagram of the included and excluded articles. RCT, randomized controlled trial; CNKI, Chinese National Knowledge Infrastructure; CBM, Chinese BioMedical Database.

antibiotics was lower than that of antibiotics alone $(\mathrm{RR}=0.35$, 95\% CI=0.13-0.97, P=0.04; Fig. 6).

Bacterial clearance rate. Bacterial clearance rates were reported in two studies $(20,22)$. The interventions were SJTs combined with LTs vs. LTs and SJTs combined with GTs vs. GTs, respectively. The interventions were classified as SJT combined with antibiotics vs. antibiotics to comprehensively evaluate the combined effect. The fixed-effects model was used for analysis $\left(\mathrm{P}=0.36, \mathrm{I}^{2}=0 \%\right)$, revealing that the bacterial clearance rate of SJT combined with antibiotics was higher than that of antibiotics alone ( $R R=1.41,95 \% \mathrm{CI}=1.09-1.84$, $\mathrm{P}=0.009$; Fig. 7).

Incidence of ADRs. A total of four studies reported on the incidence of ADRs $(20,22,27,28)$. The combined antibiotics in the four studies included LTs, GTs and OTs. The homogeneity among the studies was good $\left(\mathrm{P}=0.34, \mathrm{I}^{2}=11 \%\right)$, and the fixed-effects model was used for the meta-analysis. The results demonstrated that there was no significant difference in the incidence of ADRs between SJT combined with antibiotics and antibiotics alone $(\mathrm{RR}=0.61,95 \% \mathrm{CI}=0.32-1.17 ; \mathrm{P}=0.14$; Fig. 8).

ADRs/ADEs. In total, seven studies (710 cases) mentioned observation regarding ADRs or ADEs and three studies (23-25) reported positive results. A study (22) reported on one case of slight increase in total bilirubin, one case of slight decrease in blood leukocytes and one case of proneness to hunger in the SJT group, as well as one case of headache, one case of stomach ache and one case of elevated blood pressure in the LT group, and one case of thirst and proneness to hunger in the combined group. Another study (17) reported two cases of nausea and stomach discomfort, and two cases of mild diarrhea in the experimental group, in addition to five cases of nausea and stomach discomfort, three cases of loss of appetite and three cases of mild dysuria in the control group. In addition, one study (27) reported five cases of mild nausea and 


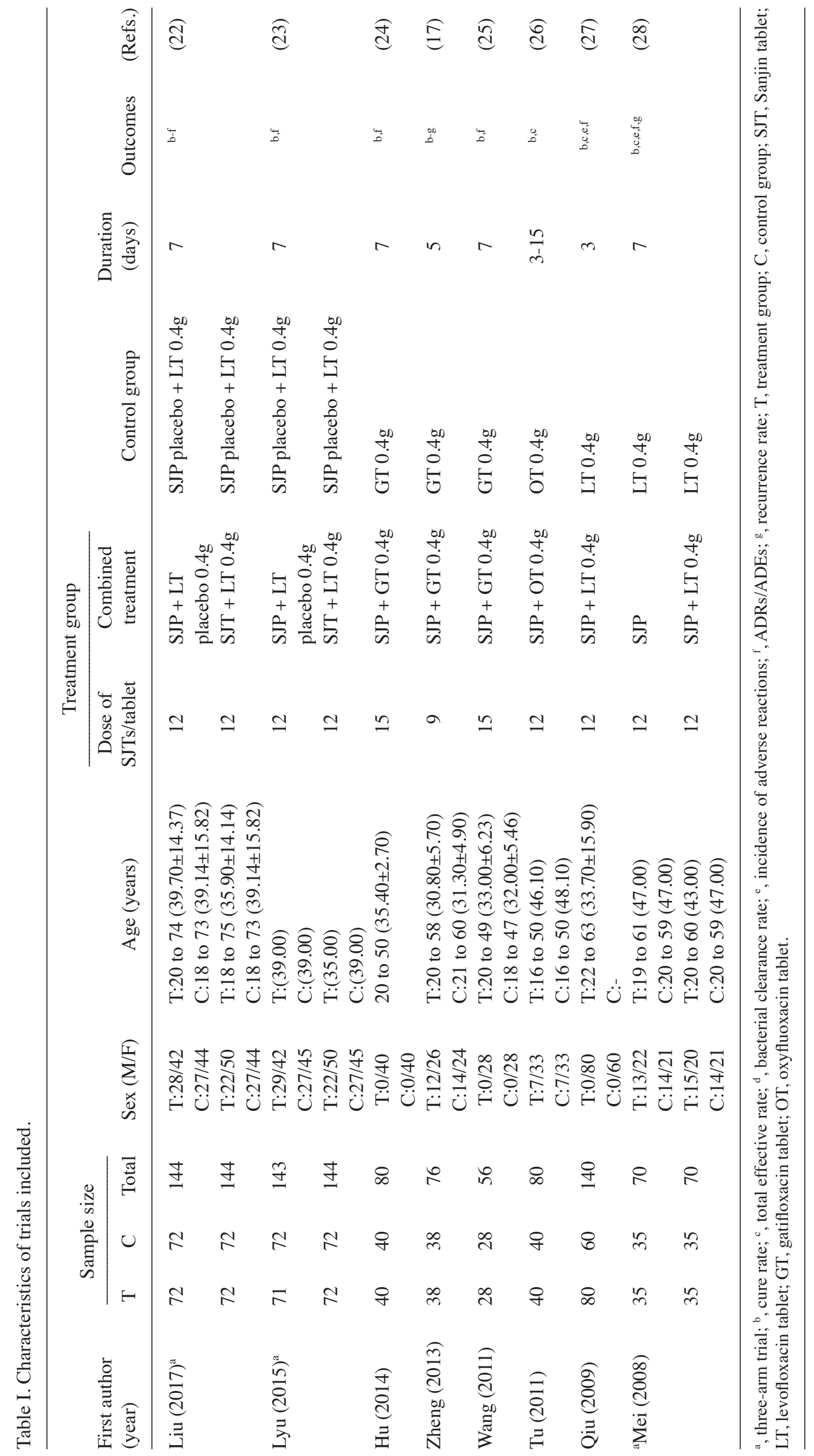




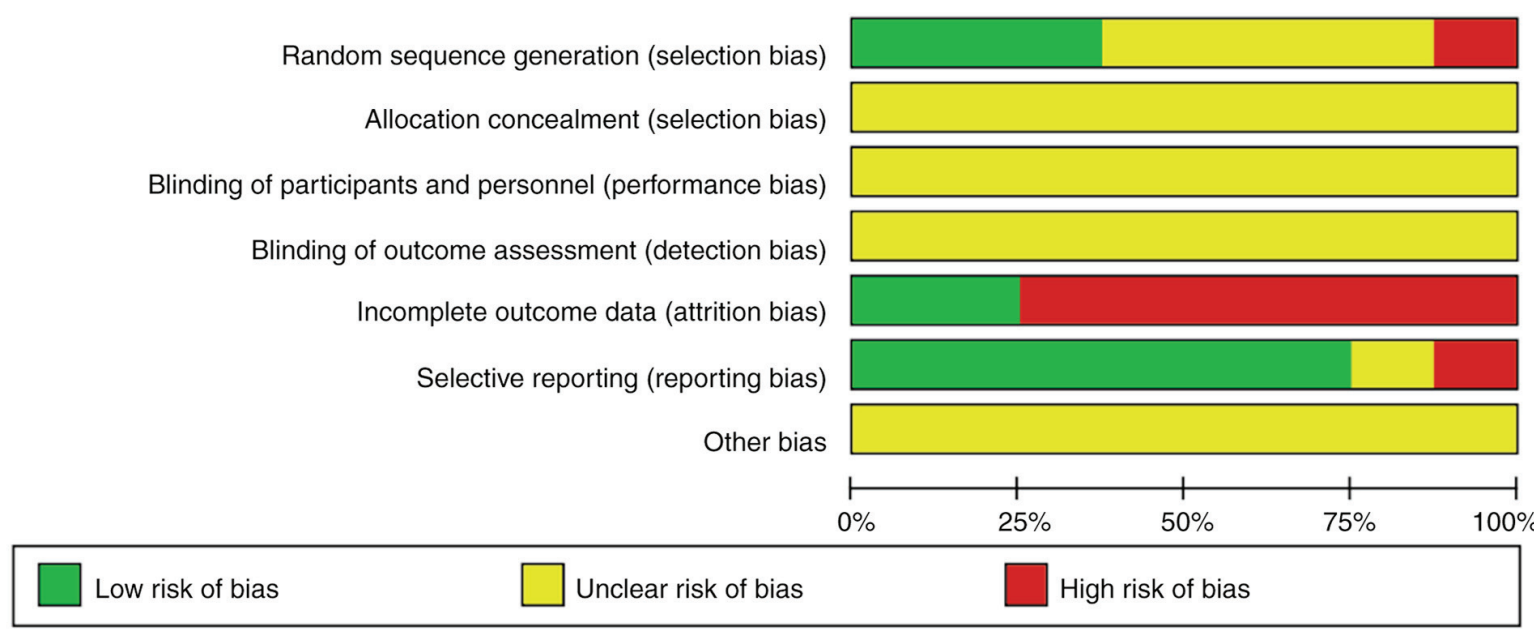

Figure 2. Risk of bias graph indicating the review authors' rating regarding the risk of bias, presented as percentages, across all of the included studies.

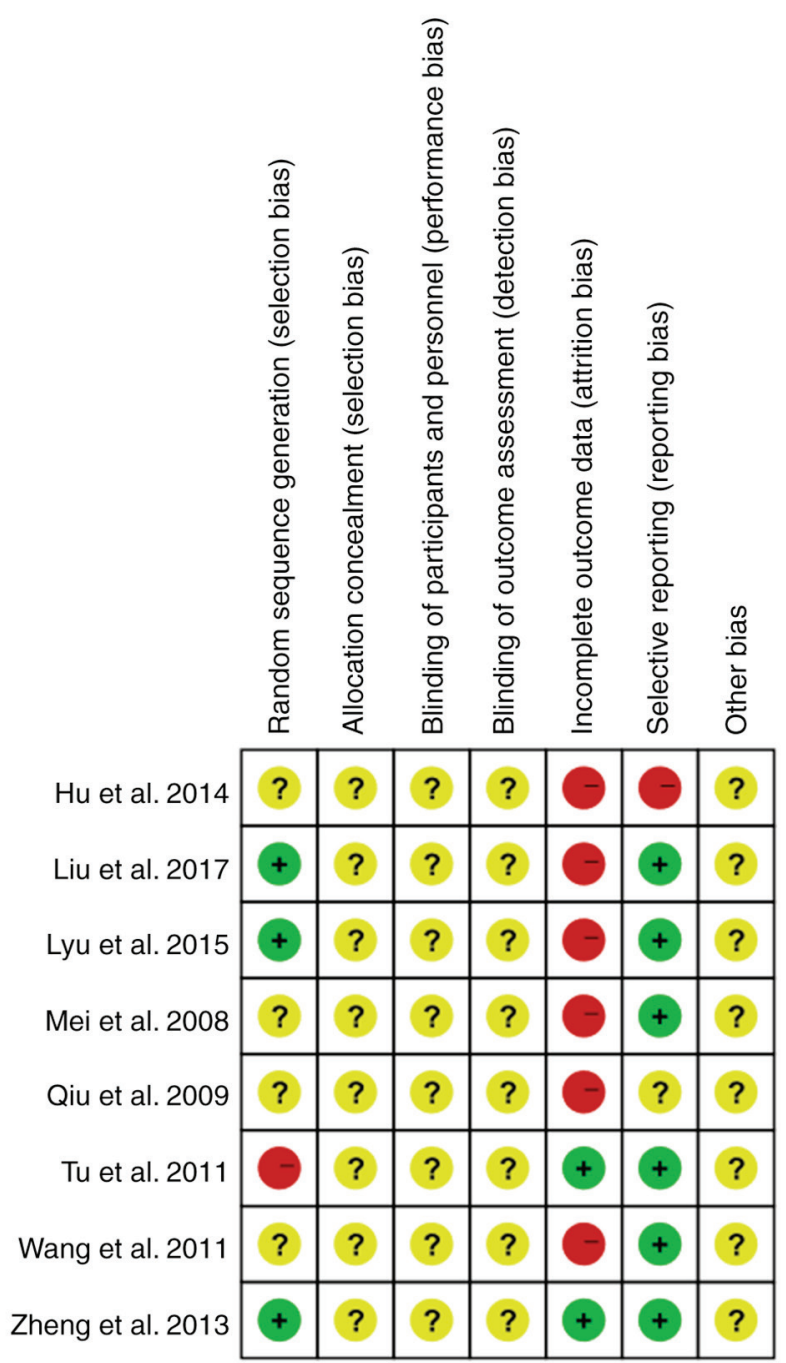

Figure 3. Risk of bias summary indicating the review authors' judgments on each risk of bias item for each included study. Green color, low risk of bias; yellow color, unclear risk of bias; red color, high risk of bias.

dizziness in the experimental and control groups, respectively. Another study (28) reported three cases of stomach discomfort and one case of skin rash in the combined treatment group, one case of stomach discomfort in the SJT group and two cases of stomach discomfort in the LT group.

Publication bias. The funnel plot was asymmetric when pooling seven trials on the cure rate (Fig. 9). The potential publication bias may be due to the high proportion of published positive results in China. All of the studies included in the present meta-analysis are written in Chinese, which may cause linguistic publication bias.

Sensitivity analysis. A sensitivity analysis of the six trials with incomplete reporting was performed $(22-25,27,28)$ using Revman software. The P-value of the overall pooled estimate changed significantly after removing one study at a time regarding four outcomes (cure rate, total effective rate, recurrence rate, incidence of adverse reactions). The results of the sensitivity analysis indicated that the sensitivity was high and the results of the meta-analysis were not stable and reliable. This suggests that the present results require confirmation using high-quality RCTs and larger samples. Clinicians should therefore exercise caution when using the present results. The P-values obtained in the sensitivity analysis for the six trials with incomplete reporting are provided in Table II.

GRADE evidence profile. The quality of evidence for the cure rate, total effective rate, recurrence rate and incidence of ADRs was very low, low, very low and low, respectively, due to the lack of randomization, blinding and allocation concealment, small sample size and publication bias, respectively. The GRADE evidence profiles are provided in Table III.

\section{Discussion}

The purpose of the present meta-analysis was to evaluate the efficacy of SJT combined with antibiotics in the treatment of ALUTIs. In order to provide accurate evidence for clinical practice, the cure rate and total effective rate were assessed. Under the same curative effect standard, combined analysis of 3 studies revealed that the cure rate of SJT combined with GT was higher than that of GT alone, while combined analysis of 4 studies indicated that the cure rate of SJT combined with LT 


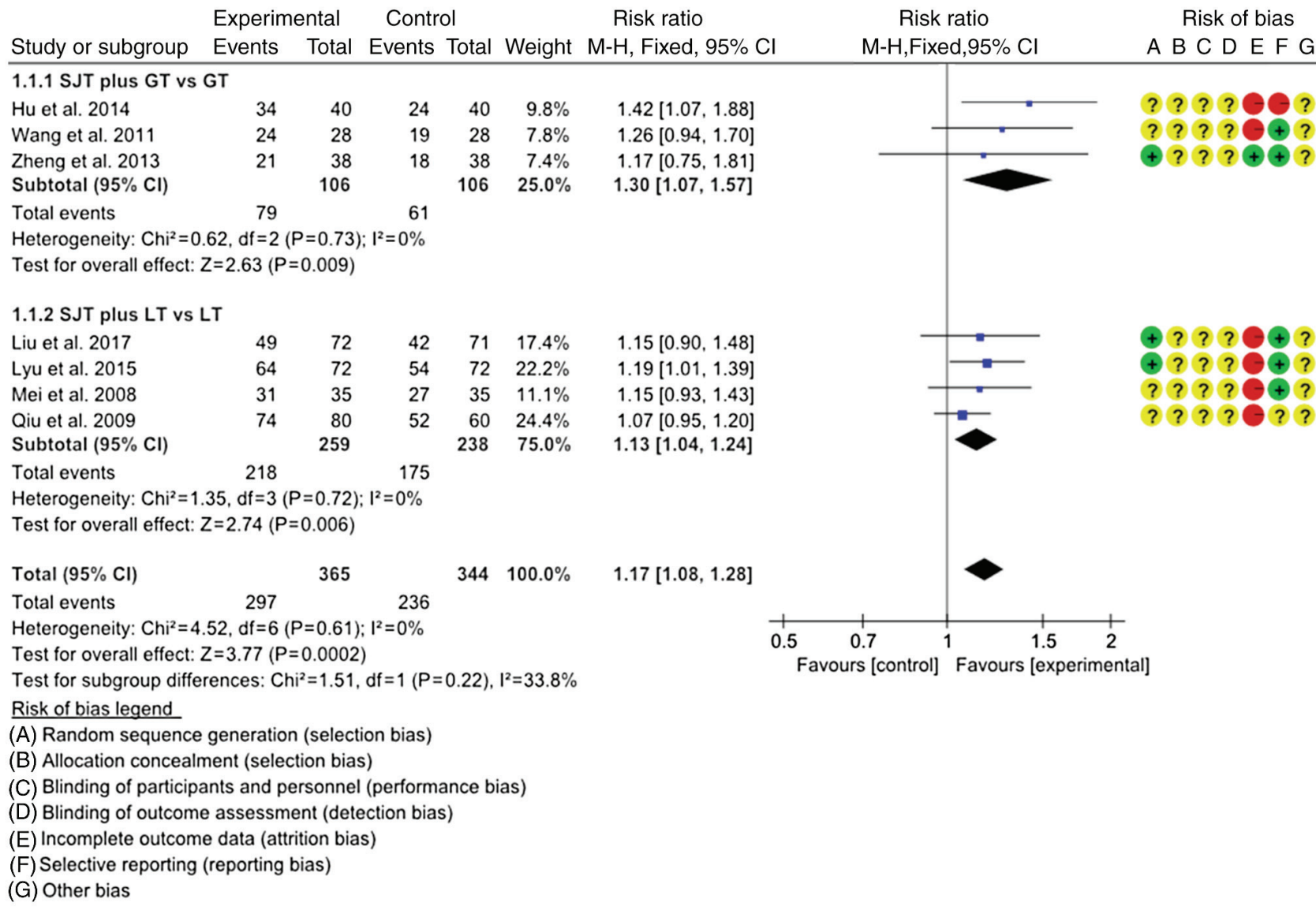

Figure 4. Forest plots of the cure rate of SJT combined with antibiotics vs. antibiotics only. Antibiotics included GT tablet and LT tablet. M-H, Mantel-Haenszel; df, degrees of freedom; SJT, Sanjin tablet; GT, gatifloxacin tablet; LT, levofloxacin tablet.

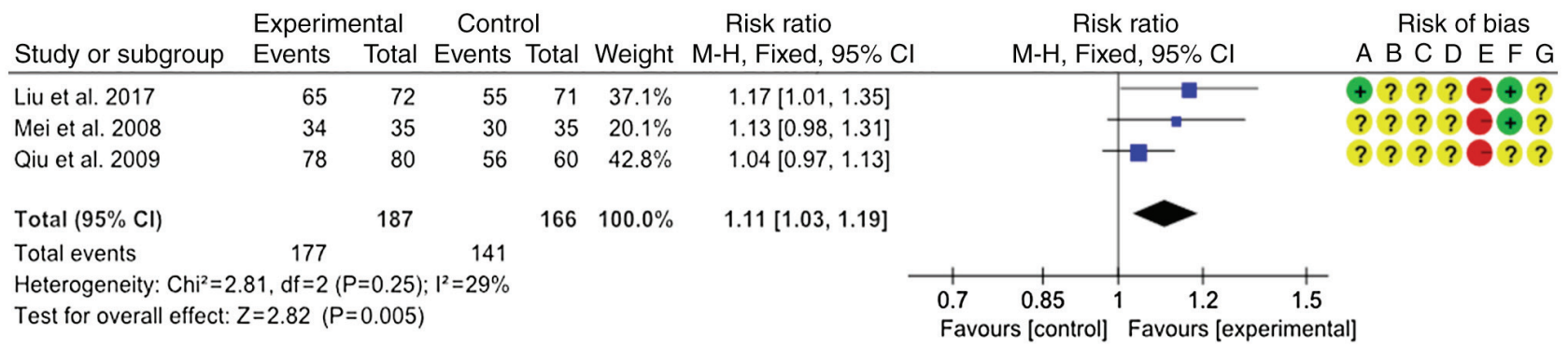

Risk of bias legend
(A) Random sequence generation (selection bias)
(B) Allocation concealment (selection bias)
(C) Blinding of participants and personnel (performance bias)
(D) Blinding of outcome assessment (detection bias)
(E) Incomplete outcome data (attrition bias)
(F) Selective reporting (reporting bias)
(G) Other bias

Figure 5. Effect of Sanjin tablet combined with levofloxacin tablets vs. levofloxacin tablets on the total effective rate. M-H, Mantel-Haenszel; df, degrees of freedom.

was higher than that of LT alone. In addition, 1 study indicated that the cure rate of SJT combined with OT was higher than that of OT alone. Combined analysis of 3 studies suggested that the total effective rate of SJT combined with LT was higher than that of LT alone and 1 study indicated that the total effective rate of SJT combined with GT was higher than that of GT alone. Combined analysis of 3 studies revealed that the recurrence rate of SJT combined with antibiotics was lower than that of antibiotics alone. Combined analysis of 2 studies indicated that the bacterial clearance rate of SJT combined with antibiotics was higher than that of antibiotics alone. The present meta-analysis demonstrated that SJT combined with antibiotics improved the clinical curative effect in the treatment of ALUTIs. Addition of SJT to antibiotics significantly 


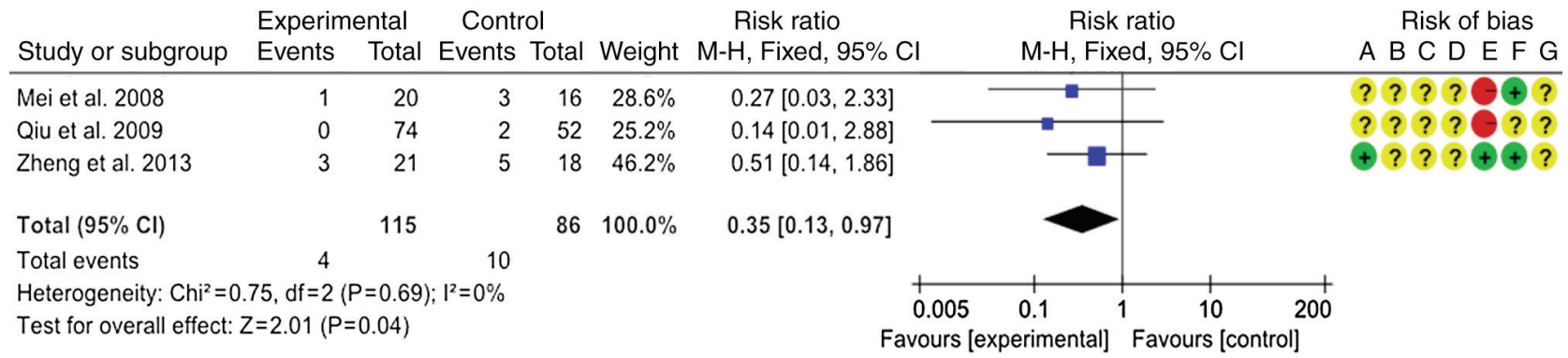

Risk of bias legend
(A) Random sequence generation (selection bias)
(B) Allocation concealment (selection bias)
(C) Blinding of participants and personnel (performance bias)
(D) Blinding of outcome assessment (detection bias)
(E) Incomplete outcome data (attrition bias)
(F) Selective reporting (reporting bias)
(G) Other bias

Figure 6. Effect of Sanjin tablet combined with antibiotics vs. antibiotics on the recurrence rate of infection. Antibiotics included gatifloxacin tablets and levofloxacin tablets. M-H, Mantel-Haenszel; df, degrees of freedom.

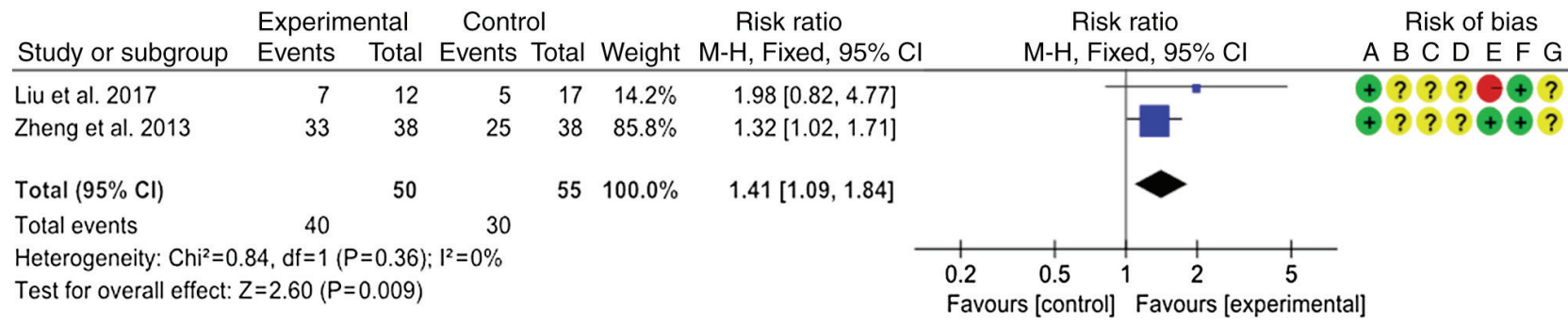
Risk of bias legend
(A) Random sequence generation (selection bias)
(B) Allocation concealment (selection bias)
(C) Blinding of participants and personnel (performance bias)
(D) Blinding of outcome assessment (detection bias)
(E) Incomplete outcome data (attrition bias)
(F) Selective reporting (reporting bias)
(G) Other bias

Figure 7. Effect of Sanjin tablets combined with antibiotics vs. antibiotics on bacterial clearance rate. Antibiotics included gatifloxacin tablets and levofloxacin tablets. M-H, Mantel-Haenszel; df, degrees of freedom.

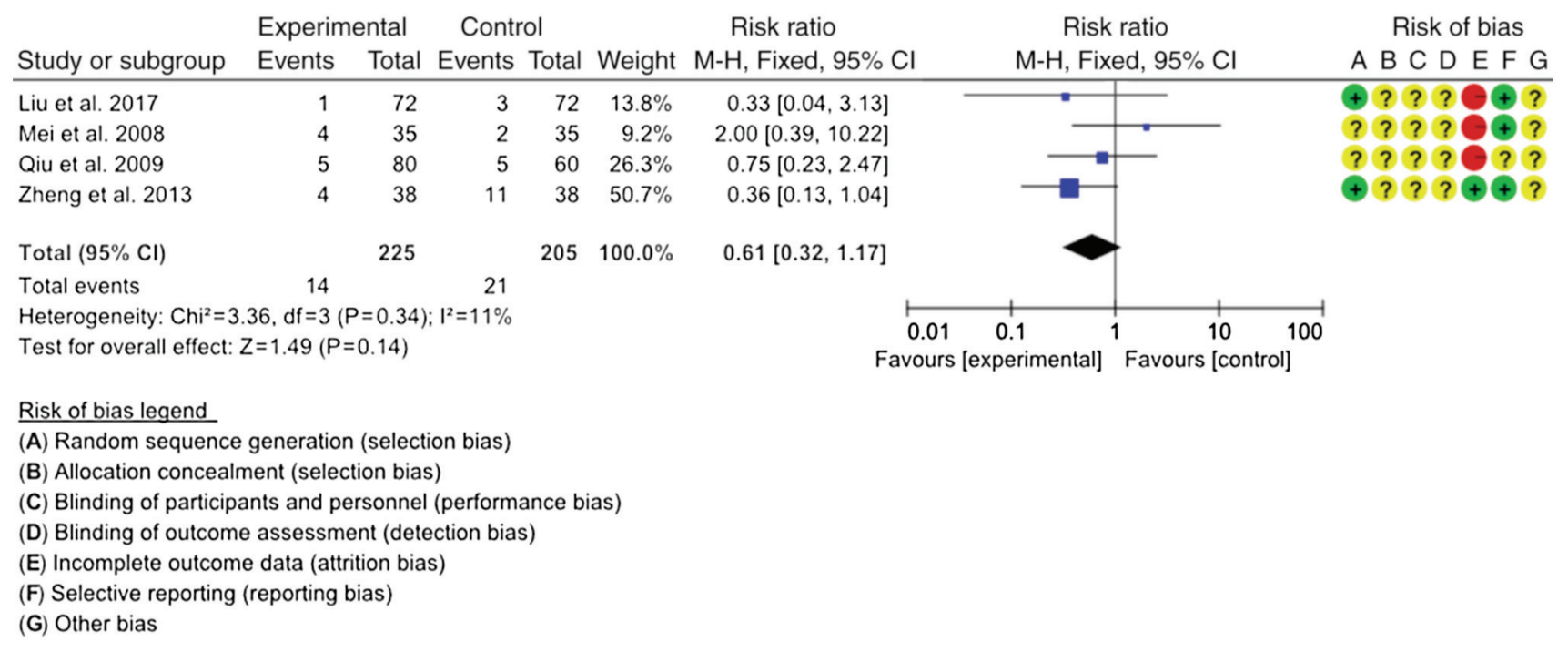

Figure 8. Effect of Sanjin tablet combined with antibiotics vs. antibiotics on the incidence of adverse reactions. Antibiotics included gatifloxacin tablets and levofloxacin tablets. M-H, Mantel-Haenszel; df, degrees of freedom. 
Table II. Sensitivity analysis of six trials with incomplete reporting.

\begin{tabular}{|c|c|c|c|}
\hline Outcome & $\begin{array}{c}\text { Study removed } \\
{[\text { first author (year)] }}\end{array}$ & P-value & $\mathrm{RR}(95 \% \mathrm{CI})$ \\
\hline Cure rate (SJT + LT vs. LT) & Liu (2017) & 0.008 & $1.13(1.03-1.23)$ \\
\hline Cure rate (SJT + LT vs. LT) & Lyu (2015) & 0.060 & $1.11(1.00-1.24)$ \\
\hline Cure rate (SJT + LT vs. LT) & Qiu (2009) & 0.010 & $1.17(1.03-1.31)$ \\
\hline Cure rate (SJT + LT vs. LT) & Mei (2008) & 0.010 & $1.13(1.02-1.25)$ \\
\hline Cure rate (SJT + GT vs. GT) & $\mathrm{Hu}(2014)$ & 0.140 & $1.22(0.94-1.58)$ \\
\hline Cure rate (SJT + GT vs. GT) & Wang (2011) & 0.030 & $1.31(1.02-1.67)$ \\
\hline Total effective rate (SJT + LT vs. LT) & Liu (2017) & 0.050 & $1.07(1.00-1.15)$ \\
\hline Total effective rate (SJT + LT vs. LT) & Qiu (2009) & 0.010 & $1.15(1.04-1.29)$ \\
\hline Total effective rate (SJT + LT vs. LT) & Mei (2008) & 0.020 & $1.10(1.02-1.19)$ \\
\hline Recurrence rate (SJT + LT vs. LT) & Mei (2008) & 0.110 & $0.38(0.12-1.22)$ \\
\hline Bacterial clearance rate (SJT + LT vs. LT) & Liu (2017) & 0.040 & $1.32(1.02-1.71)$ \\
\hline Incidence of adverse reactions (SJT + antibiotics vs. antibiotics) & Liu (2017) & 0.220 & $0.66(0.33-1.29)$ \\
\hline Incidence of adverse reactions (SJT + antibiotics vs. antibiotics) & Zheng (2013) & 0.740 & $0.87(0.37-2.02)$ \\
\hline Incidence of adverse reactions (SJT + antibiotics vs. antibiotics) & Qiu (2009) & 0.140 & $0.56(0.26-1.21)$ \\
\hline Incidence of adverse reactions (SJT + antibiotics vs. antibiotics) & Mei (2008) & 0.040 & $0.47(0.23-0.98)$ \\
\hline
\end{tabular}

Antibiotics included levofloxacin tablets (LTs), gatifloxacin tablet (GTs) and oxyfluoxacin tablets (OTs). CI, confidence interval; RR, relative ratio.

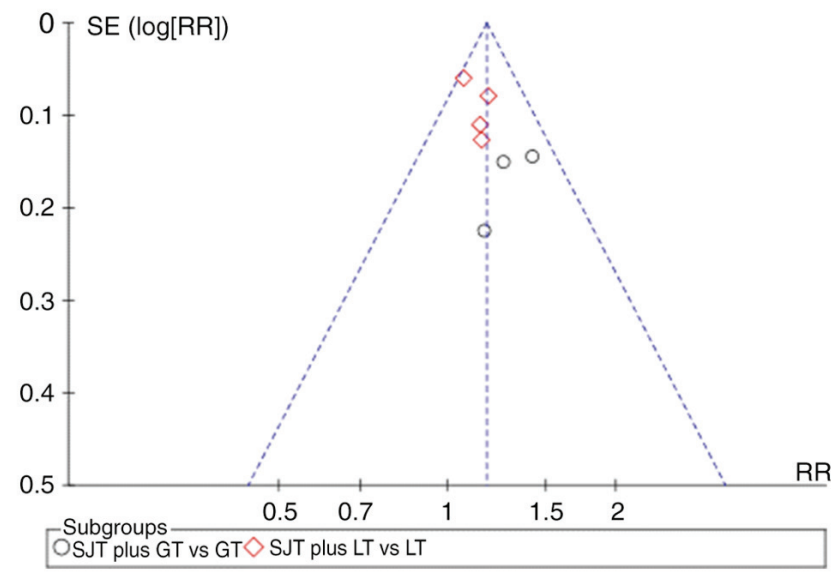

Figure 9. Funnel plot of publication bias according to the cure rate. Sanjin tablet combined with antibiotics vs. antibiotics on cure rate. Antibiotics including gatifloxacin tablets and levofloxacin tablets. SE, standard error; RR, relative risk; SJT, Sanjin tablet; GT, gatifloxacin tablet; LT, levofloxacin tablet.

improved the cure, total effective and bacterial clearance rates, and decreased the recurrence rate. However, the sensitivity analysis suggested that the stability and reliability of the results were poor. More RCTs with better consistency and fewer confounding factors are required to further verify the results, so as to provide reliable evidence for clinical practice.

The incidence of ADRs reported in the studies was summarized. The results of 4 studies suggested that there was no significant difference between SJT plus antibiotics and antibiotics alone regarding the incidence of ADRs. Thus, the addition of SJT to antibiotics may not increase the incidence of ADRs. No ADRs were reported in 3 studies, while 4 studies reported a slight increase in total bilirubin, a mild decrease in blood leukocytes, increased hunger, headache, stomach ache, elevated blood pressure, thirst, nausea, stomach discomfort, mild diarrhea and reduced appetite, mild dysuria and skin rash in the control groups. All ADRs were minor or tolerable and commonly disappeared naturally or after drug withdrawal. There were no serious ADRs or ADEs reported in any of the trials included. However, the methodological quality of the studies included in the present analysis was poor and safety requires to be further clarified by standard centralized monitoring of hospital patients.

In terms of the cure rate, this was significantly higher in the experimental group compared with that in the control group. However, there was a marked risk of bias caused by blinding, randomization, allocation concealment and publication bias. Inaccuracy due to small sample size was also present. According to the 5 degradation factors (risk of bias, inconsistency, indirectness, imprecision and reporting bias) in the GRADE system, the quality of evidence for the cure rate of SJT combined with antibiotics for ALUTI was very low and low, respectively. The total effective rate in the experimental group was higher than that in the control group; however, due to the risk of bias caused by the lack of blinding, randomization, allocation concealment and publication bias due to the small sample size, the quality of evidence for the total effective rate of SJT combined with LTs for ALUTIs decreased from high to low. Meta-analysis demonstrated that the recurrence rate of SJT combined with antibiotics was lower than that of antibiotics alone. However, the quality of evidence decreased from high to very low due to the risk of bias caused by blinding, randomization, allocation concealment, imprecision and publication bias caused by the small sample size. The quality of evidence regarding the incidence of ADRs was low due to the risk of bias caused by blinding, randomization, allocation 


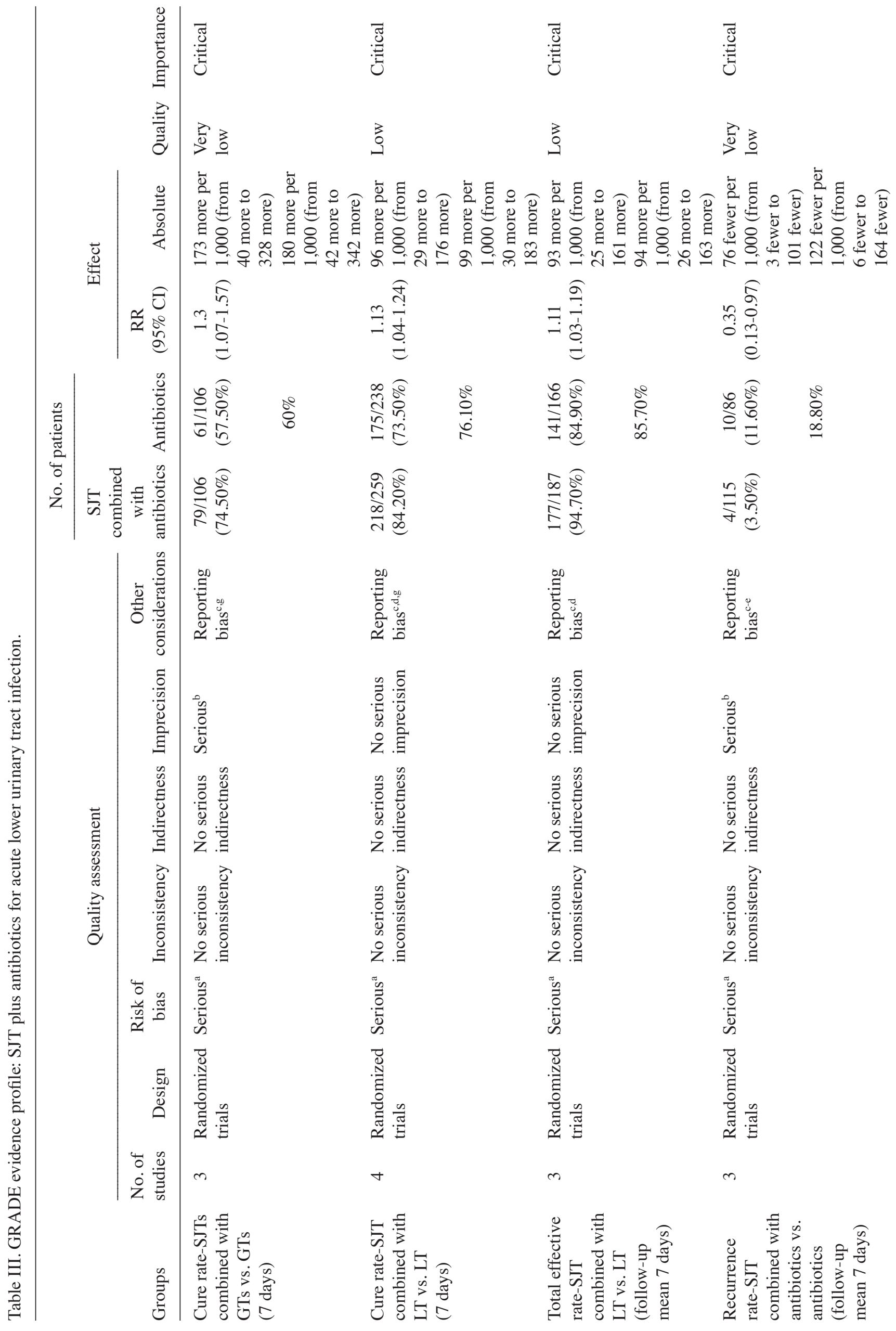




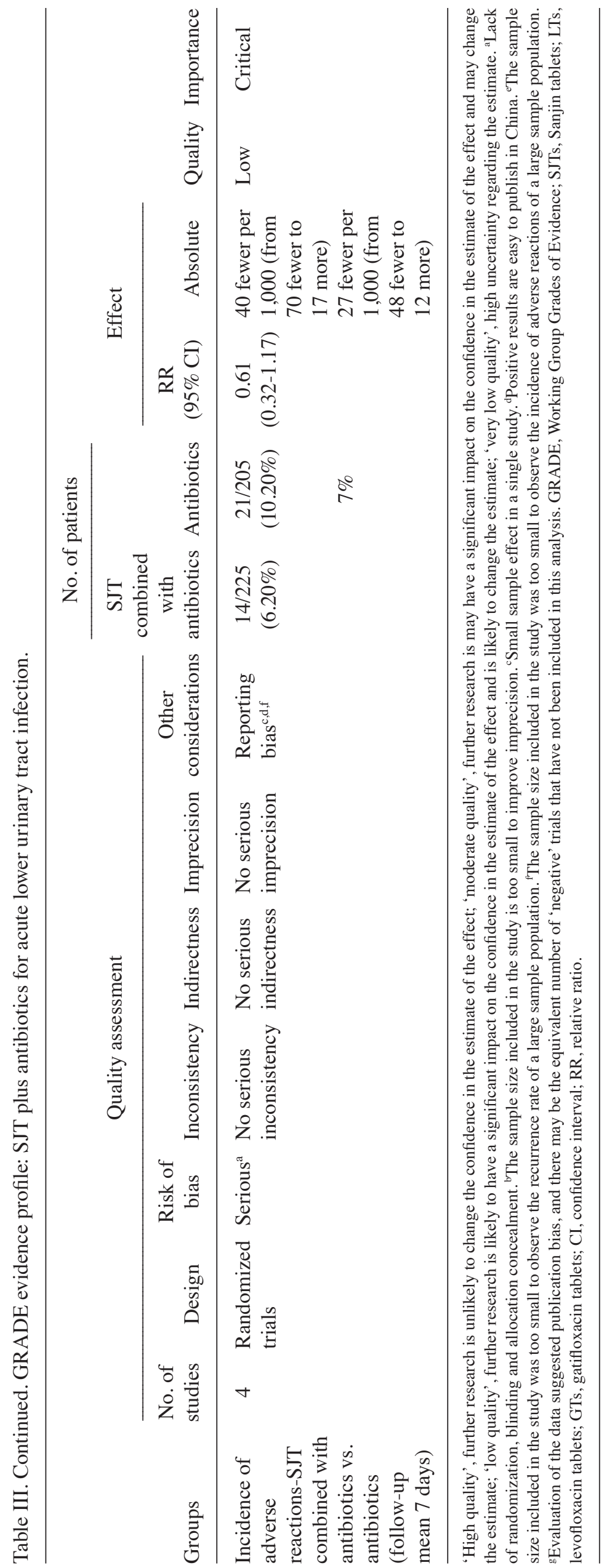


concealment and publication bias. The results of the evaluation of the quality of evidence of the above outcomes should be combined with factors including the patients' value intention and cost in order to provide recommendations, and serve as a reference or basis for clinical practice guidance.

The major limitations of the present study are as follows: i) None of the studies included reported blinding, randomization or allocation concealment, which may result in risk of bias; ii) study protocol, informed consent or ethical statements were not specified in any of the studies; iii) 6 studies reported that follow-up was performed after treatment to evaluate recurrence, but only 3 studies included follow-up data and it was not possible to evaluate the long-term effect in the cohorts of the other studies; iv) 6 studies reported on case shedding, all of which failed to perform an intentionality analysis and had incomplete reports; v) 1 study did not fully report on pre-specified outcomes and featured selective reporting; vi) no sample size estimation was reported in any of the studies included. Specifically, 3 studies had a sample size $\geq 100$ cases and 5 studies had a sample size $\leq 80$ cases. In various studies, the curative effect index was unstable and the test efficiency was low due to the small sample size. The overall methodological quality of the studies included was low. It has been suggested that large-sample, low-bias clinical RCTs should first refer to Consolidated Standards of Reporting Trials (29).

The results of the present meta-analysis suggested that, in clinical practice, addition of SJT to the use of antibiotics may be considered in order to improve the curative effect and reduce the recurrence rate in patients with ALUTIs. Due to the poor methodological quality of the studies included, the results of the present meta-analysis require to be further confirmed. The level of evidence obtained in the present study is low; thus, the expert consensus method was employed to confirm whether it may be widely used in the clinic, including the nominal group and Delphi methods. Clinicians should interpret the results of the present study with caution with regard to the actual situation and perform clinical treatments based on comprehensive consideration of evidence, expert consensus, clinical experience and the patients' preferences. In the present study, the patients were not divided into those with complex and simple LUTIs. A previous meta-analysis focused on simple LUTIs (30). In the future, the exact efficacy of SJT for complex LUTIs may be further explored.

In conclusion, the present meta-analysis demonstrated that, compared with antibiotics treatment, SJT combined with antibiotics improved the cure rate, total effective rate and bacterial clearance rate, and decreased the recurrence rate without any serious ADRs in patients with ALUTIs. However, the GRADE quality of evidence was low. Thus, additional large-sample, high-quality RCTs with a rigorous design are required to improve the quality of evidence.

\section{Acknowledgements}

Not applicable.

\section{Funding}

This study was supported by The National Key Research and Development Program of China (grant no. 2018YFC1707400).

\section{Availability of data and materials}

All data generated or analyzed during this study are included in this published article.

\section{Authors' contributions}

JL, YMX, MHS, CZ and LXW contributed to designing the search strategy. JL and MHS conducted the searches. JL, MHS and YMX performed the data extraction. JL, CZ and YMX contributed to quality assessment. All authors contributed to drafting and revising the manuscript.

\section{Ethics approval and consent to participate}

Not applicable.

\section{Patient consent for publication}

Not applicable.

\section{Competing interests}

The authors declare that they have no competing interests.

\section{References}

1. Foxman B: The epidemiology of urinary tract infection. Nat Rev Urol 7: 653-660, 2010

2. Li XS, Feng Y, Zhou X, Fan CL and Wu XY: Study on clinical distribution and drug resistance of pathogenic bacteria of urinary tract infection in inpatients in a hospital. Chin J Disinfection 36: 279-281, 2019.

3. Foxman B: Epidemiology of urinary tract infections: Incidence, morbidity, and economic costs. Dis Mon 49: 53-70, 2003.

4. Khoshnood S, Heidary M, Mirnejad R, Bahramian A, Sedighi M and Mirzaei H: Drug-resistant gram-negative uropathogens: A review. Biomed Pharmacother 94: 982-994, 2017.

5. El Bcheraoui C, Mokdad AH, Dwyer-Lindgren L, Bertozzi-Villa A, Stubbs RW, Morozoff C, Shirude S, Naghavi M and Murray CJL: Trends and patterns of differences in infectious disease mortality among US counties 1980-2014. JAMA 319: 1248-1260, 2018.

6. Bader MS, Loeb M and Brooks AA: An update on the management of urinary tract infections in the era of antimicrobial resistance. Postgrad Med 129: 242-458, 2017.

7. Wang SH, Gao YQ, Tan HG, et al: Standardization study and curative effect Analysis of Clinical treatment Scheme of Integrated traditional Chinese and Western Medicine in the treatment of Lower urinary tract infection. J Zhejiang Univ TCM 37: 1197-1200, 2013.

8. Peng YX, Liu XQ, Wen LL, et al: Antibacterial Activities of Five Chinese Medicines of Rhei Radiset Rhizoma and Their Chemical Constituents Against Multidrug-resistant Clinical Bacteria Isolates. Chin J Exp Trad Med Formulae 20: 103-107, 2014 (In Chinese).

9. Li DY, Hou Y, Zhang KY, et al: Research progress on mechanism of anti-drug resistance of traditional Chinese medicine. Chin Med Engineering 25: 16-19, 2017 (In Chinese).

10. Hou $X$ and Wang LX: Research progress of Sanjin tablets. Evaluation Ana Drug Use Chin Hospitals 16: 1148-1151, 2016.

11. National Pharmacopoeia Committee: Pharmacopoeia of the People's Republic of China. China Medical Science Press, 2015.

12. Wei XY and Lu XL: Research progress on pharmacological action of Jinyinggen. Trace Elements Health Res 34: 8081, 2017.

13. Xie Y, Hu D, Zhong C, Liu KF, Fang E, Zhang YJ, Zhou C and Tian LW: Anti-inflammatory furostanol saponins from the rhizomes of Smilax china L. Steroids 140: 70-76, 2018.

14. Zhou YL, Zhao X, Hua J, et al: Studies on the Chemical constituents and Antioxidant activity of Jinshateng. Chin J TCM 28: 1392-1396, 2013 (In Chinese). 
15. Lyu J, Xie YM, Gao Z, Shen JW, Deng YY, Xiang ST, Gao WX, Zeng WT, Zhang CH, Yi DH, et al: Sanjin tablets for acute uncomplicated lower urinary tract infection (syndrome of dampness-heat in the lower Jiao): Protocol for randomized, double-blind, double-dummy, parallel control of positive drug, multicenter clinical trial. Trials 20: 446, 2019.

16. Dong XL: Clinical efficacy and safety of Sanjin tablets combined with levofloxacin tablet in the treatment of urinary tract infection. The World's Latest Med Information Abstracts 17: 169-170, 2017.

17. Zheng HY and Hu JG: Efficacy of Sanjin tablets combined with gatifloxacin in the treatment of acute simple lower urinary tract infection. Zhejiang J Integrated Traditional Chin Western Med 23: 724-726, 2013

18. Mao X, Yao RM, Xu YH, et al: Establishment of rat model of acute urinary tract infection and its application in efficacy evaluation of traditional Chinese medicine. Pharmacol Clinic TCM 35: 177-180, 2019

19. Balshem H, Helfand M, Schünemann HJ, Oxman AD, Kunz R, Brozek J, Vist GE, Falck-Ytter Y, Meerpohl J, Norris S and Guyatt GH: GRADE guidelines: 3. Rating the quality of evidence. J Clin Epidemiol 64: 401-406, 2011.

20. Guyatt GH, Oxman AD, Sultan S, Glasziou P, Akl EA Alonso-Coello P, Atkins D, Kunz R, Brozek J, Montori V, et al: GRADE guidelines: 9. Rating up the quality of evidence. J Clin Epidemiol 64: 1311-1316, 2011.

21. Higgins J and Green SE (eds): Cochrane Handbook for Systematic Reviews of Interventions. version 5.1.0. The Cochrane Collaboration, 2011. https://handbook-5-1.cochrane. org/. Updated March, 2011.

22. Liu H, Xie JX and Xu Z: Clinical study on treatment of Acute simple bacterial Lower urinary tract infection with combination of traditional Chinese and Western Medicine. J TCM 32: 2489-2492, 2017.

23. Lyu GR and Zhan YL: Clinical observation of Sanjin tablet combined with levofloxacin in the treatment of acute simple lower urinary tract infection. Chin Med J 50: 105-107, 2015 .
24. Hu XL: Clinical analysis of Sanjin tablet combined with gatifloxacin in the treatment of acute lower urinary tract infection. Med Information 27: 659-660, 2014.

25. Wang DZ: Clinical analysis of Sanjin tablet combined with gatifloxacin in the treatment of acute lower urinary tract infection. J Med Forum 32: 164-165, 2011.

26. Tu Z and Wang T: Analysis of the efficacy of Sanjin tablet combined with ofloxacin in the treatment of acute lower urinary tract infection. China Grass-Roots Med 18: 2999-3000, 2011.

27. Qiu MS, Xu ZJ and Zhang CY: Analysis of 80 cases of female Acute Lower urinary tract infection treated by combination of traditional Chinese and Western Medicine. China Grass-Roots Med 16: 2076, 2009

28. Mei XF and Zhang CT: Clinical observation of Sanjin tablet in the treatment of acute simple lower urinary tract infection. J Modern Integration Traditional Chin Western Med 26: 4085-4086, 2008

29. Moher D, Hopewell S, Schulz KF, Montori V, Gøtzsche PC, Devereaux PJ, Elbourne D, Egger $M$ and Altman DG: CONSORT: CONSORT 2010 explanation and elaboration: Updated guidelines for reporting parallel group randomised trials. Int J Surg 10: 28-55, 2012.

30. Pu X, Zhang LY and Zhang JH: A systematic review of Sanjin tablets in the treatment of simple urinary tract infection: A randomized controlled trial. Lishizhen Med Mat Med Res 27: 1012-1014, 2016

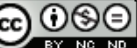

This work is licensed under a Creative Commons Attribution-NonCommercial-NoDerivatives 4.0 International (CC BY-NC-ND 4.0) License. 